\title{
Real-world assessment of the effect of impact of tumor size on pathological complete response rates in triple negative breast cancer after neoadjuvant chemotherapy
}

\author{
Bruno Henrique Rala de Paula ${ }^{1 *}$, Sanjeev Kumar ${ }^{2}$, Fabiola Marques Morosini', \\ Debora Evelyn Martins Calábria Cardoso ${ }^{1,4 * *}$, Carlos Augusto Moreira de Sousa ${ }^{1}$, Susanne Crocamo ${ }^{1}$
}

${ }^{1}$ Breast Cancer Research Unit, Clinical Research Division, Instituto Nacional de Cancer (INCA), Rio de Janeiro, Brazil; ${ }^{2}$ Cancer Research UK, Cambridge Institute, University of Cambridge, Garvan Institute of Medical Research, Sydney, Australia; ${ }^{3}$ Le Sante, Advanced Centre in Oncology, Santa Catarina, Brazil; ${ }^{4}$ Division of Oncology, Brazilian Navy, Rio de Janeiro, Brazil

Contributions: (I) Conception and design: BHR de Paula, FM Morosini, S Crocamo; (II) Administrative support: BHR de Paula, FM Morosini, DEM Calábria Cardoso, CAM de Sousa, S Crocamo; (III) Provision of study materials or patients: BHR de Paula, FM Morosini, DEM Calábria Cardoso, CAM de Sousa, S Crocamo; (IV) Collection and assembly of data: BHR de Paula, FM Morosini, CAM de Sousa, S Crocamo; (V) Data analysis and interpretation: All authors; (VI) Manuscript writing: All authors; (VII) Final approval of manuscript: All authors.

Correspondence to: Bruno Henrique Rala de Paula. Breast Cancer Research Unit, Clinical Research Division, Instituto Nacional de Cancer (INCA), Rio de Janeiro, Brazil. Email: brunobhrp@hotmail.com.

\begin{abstract}
Background: Triple negative breast cancer (TNBC) is characterized rapid tumor growth, and increased metastatic potential compared to other breast cancer subtypes. However, pathological complete response (pCR) to neoadjuvant chemotherapy (NACT) can predict patients with a better prognosis. Clinical predictors of pCR such as tumor size (TS) are controversial. This study aims to evaluate the influence of TS on achieving pCR, and the associated survival outcomes.

Methods: Medical records from 310 TNBC patients treated with NACT between 2010 and 2013 in National Cancer Institute Brazil were screened. The aim study was to examine the impact of TS on pCR. We used descriptive statistics to organize and summarize TS data and all the other variables of interest. Logistic regression has done to assess if any of these variables were associated with pCR. Survival data were extrapolated using Kaplan-Meier analysis and log-rank tests.

Results: Thirty-nine (21\%) of 187 enrolled patients achieved pCR. Median age was 48 years, $50.27 \%$ were postmenopausal, $93.03 \% \mathrm{~T} 3 / \mathrm{T} 4$ and $75.39 \%$ axillar clinical node-positive; $92.51 \%$ received an anthracycline regimen followed by a taxane. Age $>40$ years $(\mathrm{P}=0.04$, OR $0.45,95 \%$ CI, 0.20-0.95) and tumor infiltrating lymphocytes (TILs) presence ( $\mathrm{P}<0.01$, OR 3.71, 95\% CI, 1.60-8.60) were factors significantly associated with increased rates of pCR. Neither the TS (IQR: 4; P=0.22, OR 0.93, 95\% CI, 0.83-1.03) nor the other subgroups analysed demonstrated any association with achieving pCR. Median follow-up was 36 months. The 5-year OS and RFS of the study population was $71.20 \%$ and $61.10 \%$ respectively.

Conclusions: Preoperative TS did not significantly impact pCR rate in our cohort of patients receiving NACT for TNBC. Characteristics associated with higher pCR rate included TILs and age $>40$ years. In addition, pCR, was indicative of better survival outcomes.
\end{abstract}

Keywords: Neoadjuvant chemotherapy (NACT); pathological complete response (pCR); triple-negative breast cancer; tumor size (TS)

Submitted Mar 05, 2020. Accepted for publication Oct 10, 2020.

doi: $10.21037 /$ cco-20-111

View this article at: http://dx.doi.org/10.21037/cco-20-111

\footnotetext{
*, Current address: Division of Oncology, University of Cambridge, Cambridge, UK;
}

**, Current address: Division of Medicine, Brazilian Navy, Rio de Janeiro, Brazil. 


\section{Introduction}

Triple negative breast cancer (TBNC) represents a heterogeneous disease with poor prognosis and frequently larger primary tumours at diagnosis (1). Generally, neoadjuvant chemotherapy (NACT) is the standard upfront treatment option in non-metastatic tumours greater than 2 centimetres and/or node positive, with an ultimate goal of pathological complete response (pCR) to improve local outcomes as well as event-free and overall survival (OS) (2).

Standard NACT regimens continue to incorporate an anthracycline and taxane-based backbone (3). Recent evidences suggest that a dose-dense chemotherapy regimen, +/- the addition of platinum agents and immunotherapy may positively impact $\mathrm{pCR}$ rates in TNBC potentially longer-term outcomes $(4,5)$.

Efforts were undertaken in recent years to establish newer targeted therapies in TNBC. Clinical studies using poly-ADP-ribosyl polymerase (PARP) Inhibitors, novel immune checkpoint inhibitors, PI3K pathway inhibitors, cyclin-dependent kinase (CDK) 4/6 inhibitors and an antibody-drug conjugate that targets Trop-2 are being evaluated in the neoadjuvant setting to define the potential role of these drugs in early-stage disease (6).

The benefit data for these new target drugs is still getting robust, further studies to understand more accurate molecular characterization of these tumors are needed. Clinical variables may still play a significant role in treatment deciding, which may have paradoxical meaning in comparison to other breast tumor subtypes (7). An inverse association between tumor size (TS) and pCR rate is known to be mainly evident in hormone-positive and HER2-positive tumors treated with NACT $(8,9)$. However, the literature is not consistent regarding TNBC (10-12). In real-world studies, pCR has been reported even in high tumor burden populations, frequently observed in developing countries (13).

In this study, we retrospectively explore the influence of TS on the rate of pCR and the associated survival outcomes in a real-word cohort of patients with TNBC treated with NACT. We present the following article in accordance with the STROBE reporting checklist (available at http://dx.doi. org/10.21037/cco-20-111).

\section{Methods}

The study was conducted in accordance with the
Declaration of Helsinki (as revised in 2013). The study was approved by the institutional Ethics Committee board of the Instituto Nacional de Cancer in 29/04/2016 (report number 54489016.9.0000.5274) with exemption from obtaining informed consent due to the methodology nature of the study.

A retrospective analysis was carried out on a cohort of patients treated in Instituto Nacional de Cancer from January 2010 to December 2013. Included patients were $\geq 18$ years, with histological confirmation of invasive breast cancer, stage II or III, estrogen receptor (ER), progesterone receptor (PR), and human epidermal growth factor receptor-type 2 (HER2) negative (TNBC) who received NACT. Patients with bilateral breast cancer or concurrently diagnosed non-TNBC and who declined to receive definitive breast surgery were excluded.

The patient and tumor variables collected for analysis were age, histological subtype, body mass index (BMI), menopausal status, cancer stage, pre-treatment TS, tumor infiltrating lymphocytes (TILs) in the pre-treatment tumor biopsy, the NACT regimen, pathological response and date of relapse, death and/or loss to follow up.

Histological confirmation of invasive carcinoma followed ASCO/CAP guidelines and TILs presence $(>10 \%)$ per the International TILs Working Group $(14,15)$. The clinical TS was measured in centimetres; ER status, PR status and HER2 status were determined by standard immune-histochemical methods. Tumors with $<1 \%$ positive cells were considered to have a negative receptor status. HER2 status was recorded as negative if there was $1+$ staining. HER2 status was confirmed by fluorescence in situ hybridization (FISH) if $2+$ immunohistochemical staining was present according to American Society of Clinical Oncology/College of American Pathologists HER2 testing guidelines at the time of diagnosis (16). We used the America Cancer Society classification to define BMI ranges.

We defined conservative breast surgery as lumpectomy, segmental resection, and quadrantectomy with lymphadenectomy or sentinel lymph node sampling, and radical breast surgery was defined as total removal of mammary gland with or without skin-sparing, with lymphadenectomy or sentinel lymph node sampling.

pCR definition was the absence of invasive breast carcinoma both in the breast and axillary lymph nodes in the surgical excision specimen (ypT0/is/ypN0). Relapsefree survival (RFS) was considered from histological confirmation date to the evidence of relapse/death from 
any cause or censored event, and OS from the histological confirmation to the day of death by any cause or censored event.

\section{Statistical analysis}

Descriptive and demographic data were summarized by medians or proportions; the TS was expressed by continuous variable as median and interquartile range (IQR) and was represented by tables. Survival curves were constructed using the Kaplan-Meier method and compared using the log-rank test to obtain $\mathrm{P}$ values in the univariate analysis.

The analysis of descriptive and demographic factors that were associated with complete response was performed using logistic regression to obtain an odds ratio (OR), confidence intervals $(\mathrm{CI})$ and $\mathrm{P}$ values in each univariate logistic model. The analyses were considered statistically significant when $\mathrm{P}$ values obtained were less than 0.05 .

Stratification of the pCR in subgroups of interest by TS are shown in boxplots to evaluate graphically if there were differences between these groups. The analyses were performed using R-Studio (R version 3.3.1), packages survival, stats and ggplot2.

\section{Results}

From the 310 TNBC patients identified between January 2010 to December 2013, 187 met the inclusion criteria. Main reason for exclusion criteria was adjuvant treatment and metastasis diagnose. The median age was 48 years and BMI $28.29 \mathrm{~kg} / \mathrm{m}^{2}$. Invasive ductal carcinoma was evident in $181(96.79 \%)$ patients. The median TS was $8.0 \mathrm{~cm}$, with clinical T3 or T4 tumour identified in 175 patients (93.58\%). A total of 139 patients (74.33\%) had positive axillary involvement. Most patients received anthracycline followed by taxane chemotherapy (92.51\%). Only four patients $(2.13 \%)$ underwent conservation surgery and the majority received mastectomy. TILs were present in 79 patients $(42.93 \%)$. The baseline characteristics are shown in more detail in Table 1.

pCR was reported in 39 patients (21\%). The following baseline clinical and pathological factors were found to be significantly associated with the odds of experiencing pCR based on univariable binary logistic regression analyses (Table 2): age higher than 40 years $(\mathrm{P}=0.04$, $\mathrm{OR} 0.45,95 \%$ CI, 0.20-0.95) and the presence of TILs $(\mathrm{P}<0.01$, OR 3.71, 95\% CI, 1.60-8.60).
Neither the TS, (IQR: 4; $\mathrm{P}=0.22$, OR 0.93, 95\% CI, 0.83-1.03) (Figure 1) nor any of the other subgroups analysed correlated with differences in pCR rate (Table 2).

The median follow-up was 36 months. At the time of the data analysis, the median RFS (relapse free survival) had not yet been reached. The 5 -year OS rate and RFS rate of the study population was respectively $71.20 \%$ and $61.10 \%$ (Figure 2A,B).

Patients who achieved pCR gained significant benefit in terms of OS (Log-rank $=13.35, \mathrm{P}=0.001)$ and RFS (Log-rank $=15.61, \mathrm{P}<0.001)$, compared to those who did not achieve pCR (Figure $3 A, B$ ).

\section{Discussion}

This retrospective analysis illustrates a real-world population of patients with TBNC who received anthracycline and taxane-based NACT in the public healthcare system of a developing country.

The median tumour size of $8.0 \mathrm{~cm}$ is double the median size reported by a pooled analysis of clinical trials (CT) performed by von Minckwitz et al., 2012 (17) and rate of T3 or T4 tumours is three times higher (Table 1). Although this might be explained by distinct inclusion criteria between CT and clinical practice (CP), it probably justifies our low rates of conservation surgeries $(2.13 \%)$, tenth times lower than the $23 \%$ achieved in studies from the afore mentioned NSABP group (17).

Thirty-nine patients $(21 \%)$ achieved pCR. Similar rates have been reported for the same chemotherapy regimen and nationality (17-19). In a multi-centre, real-world study from Italy, Gamucci et al. 2018 (20), showed double the pCR rates with similar chemotherapy regimens (42.6\%). However, this discrepancy could be justified by their limited number of node positive patients $(19.7 \%$ versus $74.39 \%$ in our cohort) and their frequent administration of concomitant anthracycline-taxane chemotherapy $(56.3 \%$ versus none of our patients).

The median $8 \mathrm{~cm}$ TS did not predict pCR (IQR: 4; $\mathrm{P}=0.22$, OR $0.93,95 \% \mathrm{CI}, 0.83-1.03$ ) in our studied population, in accordance with other published patient cohorts (8-10). Due to the high heterogeneity of TNBC and how this population is evaluated in CT, most pCR clinical predictors studied did not achieve the necessary levels of positive and negative predictive value to be implemented (21).

This suggests that other factors should be taken into account to predict tumor response than TS, such as intrinsic 
Table 1 Patient and tumor baseline characteristics

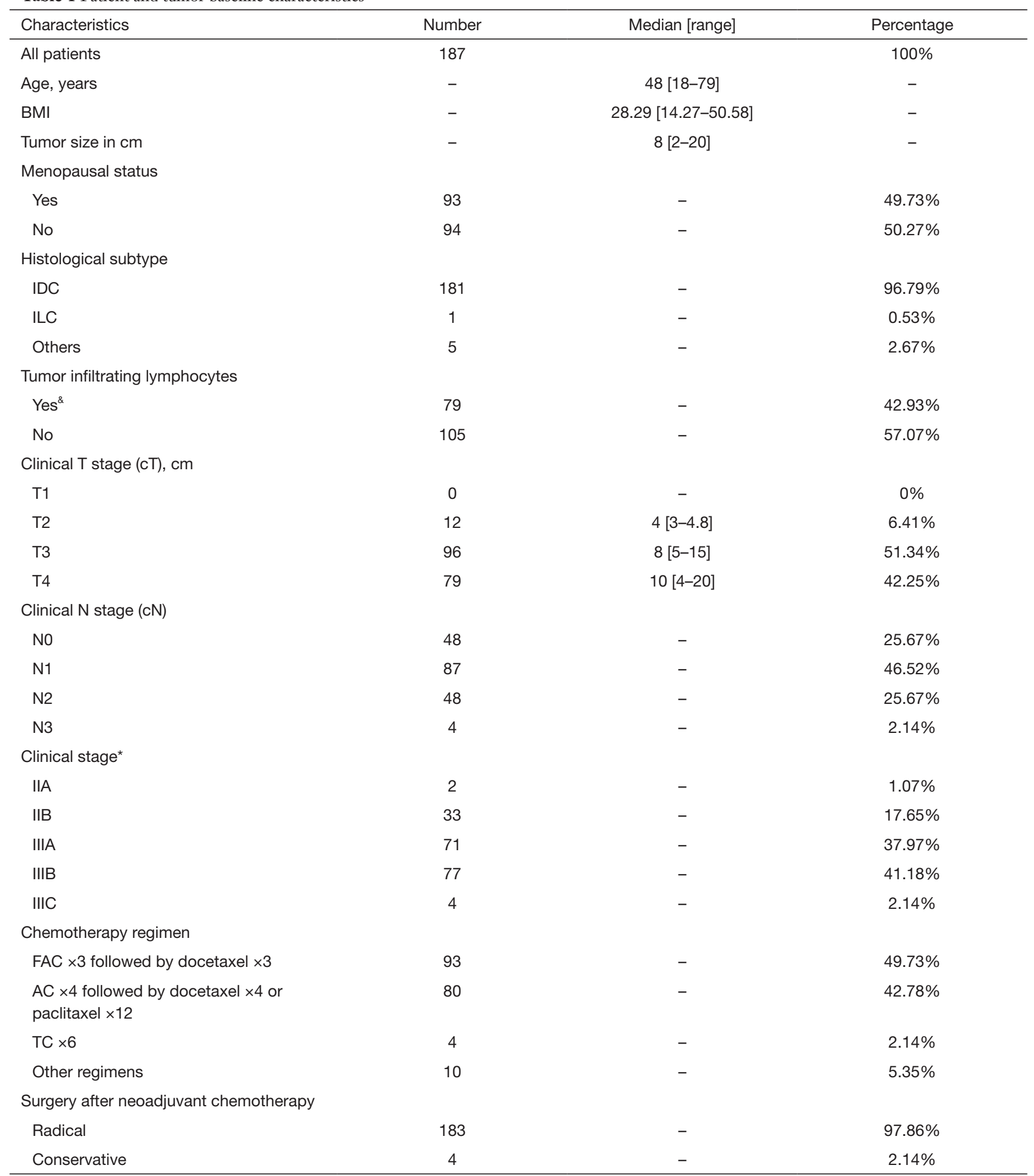

\&, more than 10\%. ${ }^{*}$, TNM classification according to the International Union Against Cancer. IDC, invasive ductal carcinoma; ILC, invasive lobular carcinoma; BMI, body mass index; FAC, 5-fluorouracil, doxorubicin and cyclophosphamide; AC, doxorubicin and cyclophosphamide; TC, docetaxel and cyclophosphamide. 
Table 2 Association of baseline factors with pCR at surgery on univariate and multivariate analysis

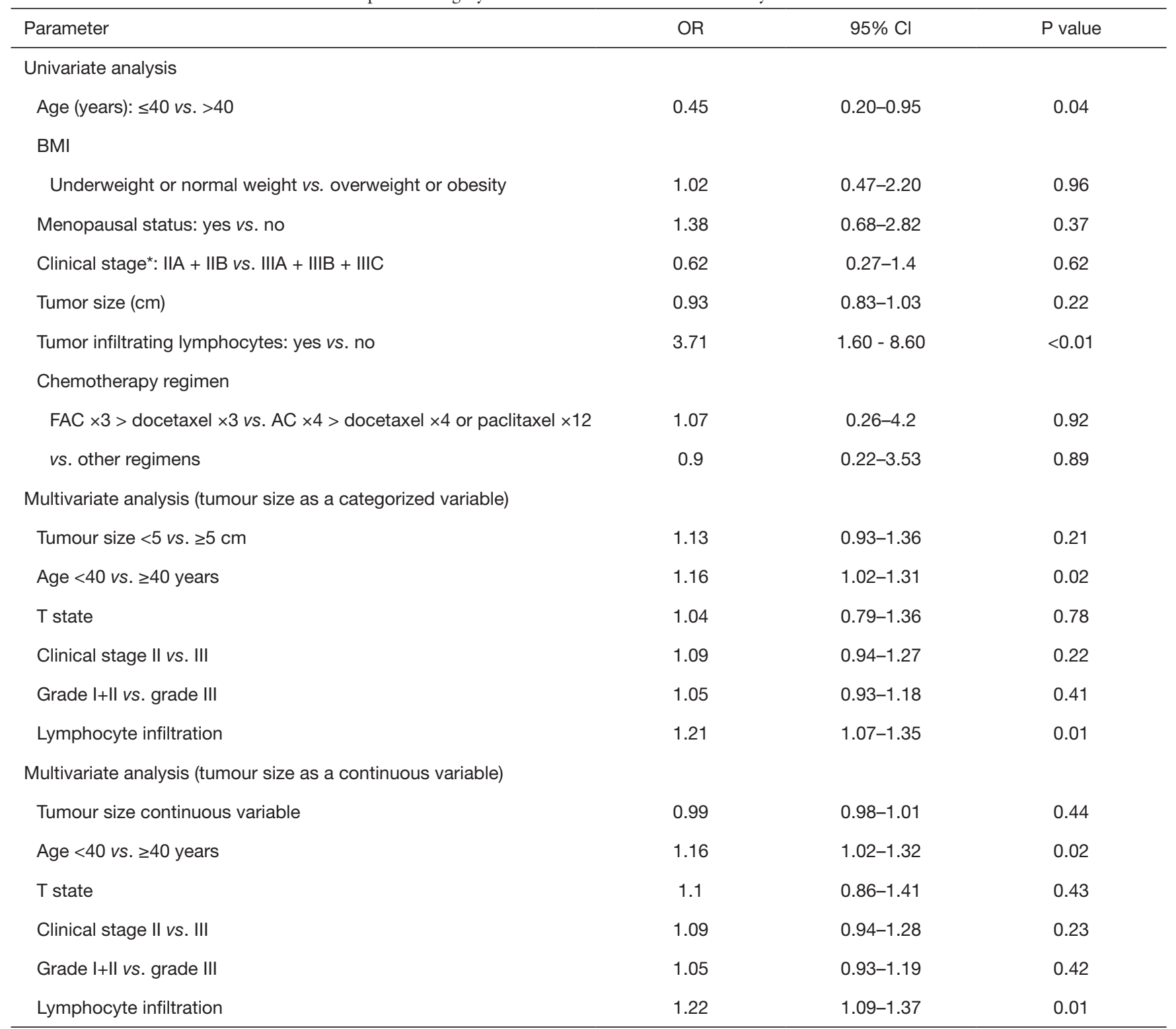

*, TNM classification according to the International Union Against Cancer. BMI, body mass index; FAC, 5-fluorouracil, doxorubicin and cyclophosphamide; AC, doxorubicin and cyclophosphamide; TC, docetaxel and cyclophosphamide; pCR, pathologic complete response; $I Q R$, interquartile range; $\mathrm{Cl}$, confidence interval; OR, odds ratio. 
molecular subtypes (22). However in an unexpected comparison to other studies, our results showed that patients over 40 years $(\mathrm{P}=0.04$, OR $0.45,95 \%$ CI, $0.20-0.95)$ were more likely to reach pCR (23).

The best molecular evidence currently used has shown a correlation with increased pCR rate in TNBC and high levels of TILs before NACT (24). Our results corroborate with this statement, where TILs presence was found to be significantly associated with an increased rate of pCR $(\mathrm{P}<0.01$, OR $3.71,95 \% \mathrm{CI}, 1.60-8.60)$. Despite this, TILs is not a proven biomarker in isolation, as there are reports of patients with low levels of TILs who still achieve pCR (24).

The pCR rate in the literature varies from more than $50 \%$ in studies with newer strategies such as

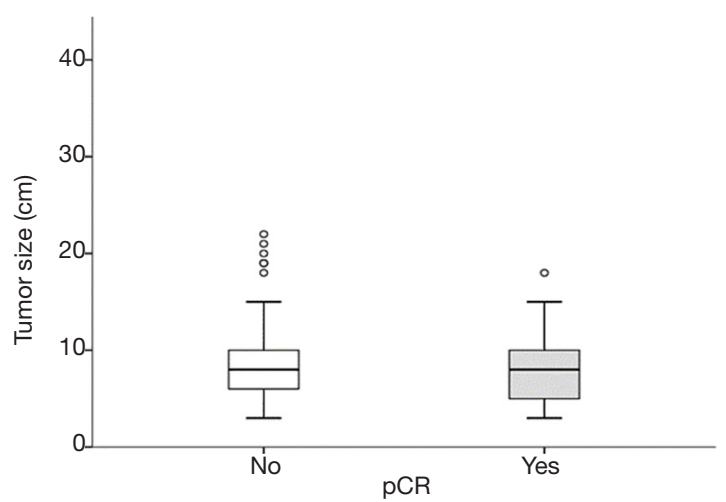

Figure 1 Boxplot of tumor size distribution between patients who reached (grey) and did not reach (white) pCR. pCR, pathological complete response. immunotherapy $(25,26)$, dose-dense chemotherapy with platinum agents' and/or bevacizumab studies (7,27-34) and parp-inhibitors $(35,36)$ to around $25 \%$ in robust, pivotal trials of sequential anthracycline taxane regimens $(16,18)$. Additionally, published studies include a very limited proportion of $\mathrm{T} 4$ tumours and some still use the historical separation of pCR in breast and axilla (32).

The importance of total dose-intensity chemotherapy delivered for breast cancer in the adjuvant setting has been widely recognised for more than 20 years (37), and the preferred regimen as NACT in TNBC in the last decade has been anthracycline and taxane-containing regimens. Our study is consistent with a metanalysis published in 2014 by Wu et al. (38), reporting a higher pCR rate with administration of chemotherapy regimens containing 6 or more cycles, however, no difference in $\mathrm{pCR}$ rate between different anthracycline and taxane containing regimens.

This is a retrospective study with limitations inherent to bias control and confounding factors. Nevertheless, the 5 -year period of observation and the fact that data has been derived from a cohort of unselected "real world" represent the major strengths of our work, illustrating that the size of the primary tumor seem does not impact directly on the rate of pCR with NACT administration in TNBC.

Some studies have been carried out in order to determine molecular predictive factors of $\mathrm{pCR}$, but without definitive success. It seems to us that for such a complex molecular subtype of breast cancer, the best method moving forward is to consider a focus on the tumor microenvironment, by understanding the communication between the extracellular matrix and surrounding cells (39). This strategy may enable
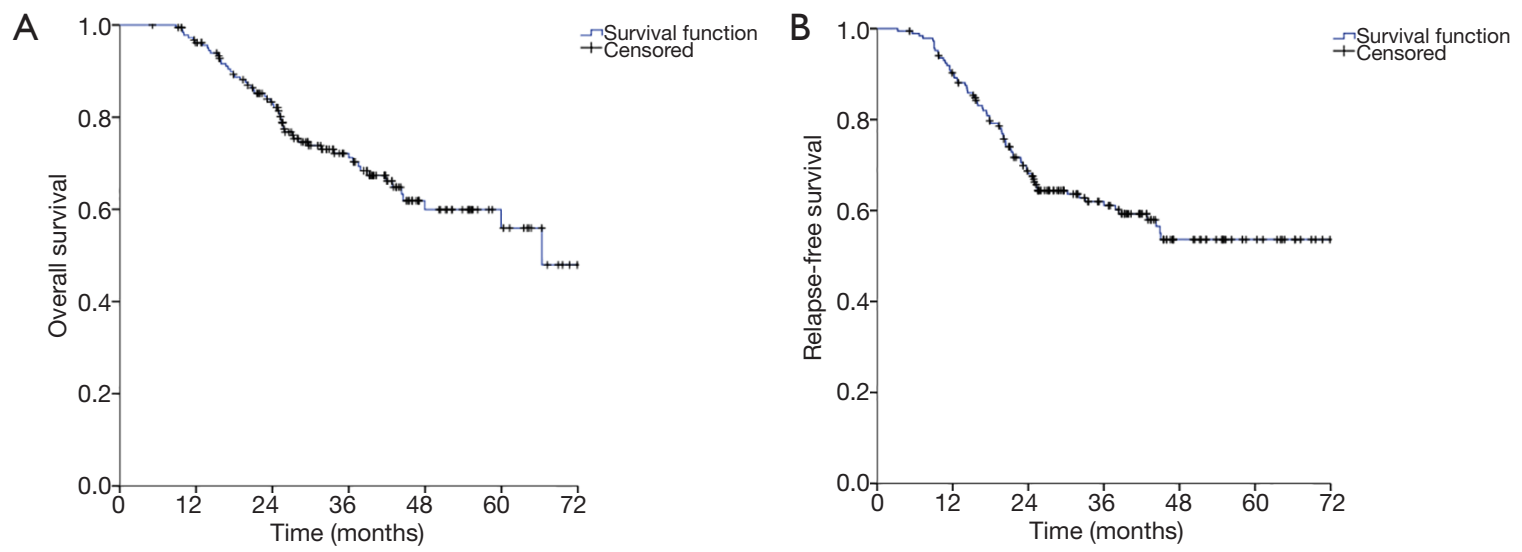

Figure 2 Kaplan-Meier survival curves of the entire TNBC study population treated with NACT. (A) Overall survival curve; (B) relapse free survival curve. TNBC, triple negative breast cancer; NACT, neoadjuvant chemotherapy. 

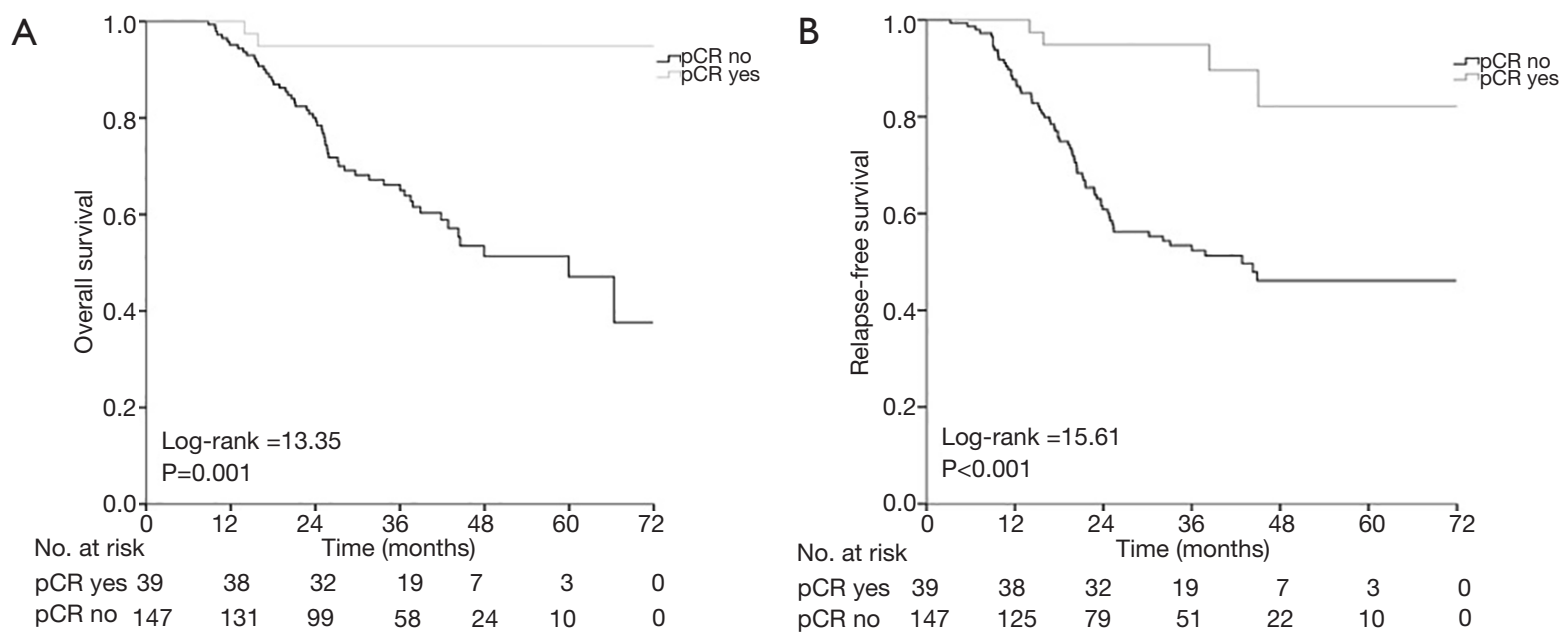

Figure 3 Impact of pathological response on survival. (A) Overall survival curves, log rank test value and P value between patients who have achieved pCR and who have not achieved. (B) Relapse-free survival curves, log rank test value and $\mathrm{P}$ value between patients who have achieved pCR and who have not achieved. pCR, pathological complete response.

identification of new biomarkers or targets in stromal components to respectively predict clinical outcomes and guide therapy in TNBC (40).

\section{Conclusions}

Preoperative TS did not impact pCR rate in our 'real world' cohort of patients, however achievement of pCR proved to be a solid surrogate biomarker of survival outcomes in our TNBC population. Better predictive tools and intrinsic molecular evaluation are ungently awaited in TNBC to help in the decision to escalate, de-escalate treatment or to incorporate new targeted therapies into the management of this complex tumor subtype.

\section{Acknowledgments}

We acknowledge the patients from National Cancer Institute Brazil and daily efforts of staff to contribute for the improvement of breast cancer care.

Funding: None.

\section{Footnote}

Reporting Checklist: The authors have completed the STROBE reporting checklist. Available at http://dx.doi. org/10.21037/cco-20-111
Data Sharing Statement: Available at http://dx.doi. org/10.21037/cco-20-111

Conflicts of Interest: All authors have completed the ICMJE uniform disclosure form (available at http://dx.doi. org/10.21037/cco-20-111). The authors have no conflicts of interest to declare.

Ethical Statement: The authors are accountable for all aspects of the work in ensuring that questions related to the accuracy or integrity of any part of the work are appropriately investigated and resolved. The study was conducted in accordance with the Declaration of Helsinki (as revised in 2013). The study was approved by the institutional Ethics Committee board of the Instituto Nacional de Cancer in 29/04/2016 (report number 54489016.9.0000.5274) with exemption from obtaining informed consent due to the methodology nature of the study.

Open Access Statement: This is an Open Access article distributed in accordance with the Creative Commons Attribution-NonCommercial-NoDerivs 4.0 International License (CC BY-NC-ND 4.0), which permits the noncommercial replication and distribution of the article with the strict proviso that no changes or edits are made and the original work is properly cited (including links to both the 
formal publication through the relevant DOI and the license). See: https://creativecommons.org/licenses/by-nc-nd/4.0/.

\section{References}

1. Brewster AM, Chavez-MacGregor M, Brown P. Epidemiology, biology, and treatment of triple-negative breast cancer in women of African ancestry. Lancet Oncol 2014;15:e625-34.

2. von Minckwitz G, Loibl S, Maisch A, et al. Lessons from the neoadjuvant setting on how best to choose adjuvant therapies. Breast 2011;20 Suppl 3:S142-5.

3. Gradishar W, Salerno KE. NCCN guidelines update: breast cancer. J Natl Compr Canc Netw 2016;14:641-4.

4. Omarini C, Guaitoli G, Pipitone S, et al. Neoadjuvant treatments in triple-negative breast cancer patients: where we are now and where we are going. Cancer Manag Res 2018;10:91-103.

5. Sxther NH, Skuja E, Irmejs A, et al. Platinum-based neoadjuvant chemotherapy in BRCA1-positive breast cancer: a retrospective cohort analysis and literature review. Hered Cancer Clin Pract 2018;16:9.

6. McCann KE, Hurvitz SA, McAndrew N. Advances in Targeted Therapies for Triple-Negative Breast Cancer. Drugs 2019;79:1217-30.

7. McAndrew N, DeMichele A. Neoadjuvant Chemotherapy Considerations in Triple-Negative Breast Cancer. J Target Ther Cancer 2018;7:52-69.

8. Cortazar P, Zhang L, Untch M, et al. Pathological complete response and long-term clinical benefit in breast cancer: the CTNeoBC pooled analysis. Lancet 2014;384:164-72.

9. Baron P, Beitsch P, Boselli D, et al. Impact of tumor size on probability of pathologic complete response after neoadjuvant chemotherapy. Ann Surg Oncol 2016;23:1522-9.

10. Goorts B, van Nijnatten TJ, de Munck L, et al. Clinical tumor stage is the most important predictor of pathological complete response rate after neoadjuvant chemotherapy in breast cancer patients. Breast Cancer Res Treat 2017;163:83-91

11. Nwaogu IY, Fayanju OM, Jeffe DB, et al. Predictors of pathological complete response to neoadjuvant chemotherapy in stage II and III breast cancer: the impact of chemotherapeutic regimen. Mol Clin Oncol 2015;3:1117-22.

12. Gass P, Lux MP, Rauh C, et al. Prediction of pathological complete response and prognosis in patients with neoadjuvant treatment for triple-negative breast cancer. BMC Cancer 2018;18:1051.

13. Luz P, Dias D, Fortuna A, et al. How shall we treat locally advanced triple negative breast cancer? F1000Res 2019;8:1649.

14. Hammond ME, Hayes DF, Dowsett M, et al. American Society of Clinical Oncology/College of American Pathologists guideline recommendations for immunohistochemical testing of estrogen and progesterone receptors in breast cancer (unabridged version). Arch Pathol Lab Med 2010;134:e48-72.

15. Salgado R, Denkert C, Demaria S, et al. The evaluation of tumor-infiltrating lymphocytes (TILs) in breast cancer: recommendations by an International TILs Working Group 2014. Ann Oncol 2015;26:259-71.

16. Wolff AC, Hammond ME, Schwartz JN, et al. American Society of Clinical Oncology/College of American Pathologists guideline recommendations for human epidermal growth factor receptor 2 testing in breast cancer. Arch Pathol Lab Med 2007;131:18-43.

17. von Minckwitz G, Untch M, Blohmer JU, et al. Definition and impact of pathologic complete response on prognosis after neoadjuvant chemotherapy in various intrinsic breast cancer subtypes. J Clin Oncol 2012;30:1796-804.

18. Mamounas EP. NSABP Protocol B-27. Preoperative doxorubicin plus cyclophosphamide followed by preoperative or postoperative docetaxel. Oncology (Williston Park) 1997;11:37-40.

19. Gonçalves H Jr, Guerra MR, Duarte Cintra JR, et al. Survival Study of Triple-Negative and Non-TripleNegative Breast Cancer in a Brazilian Cohort. Clin Med Insights Oncol 2018;12:1179554918790563.

20. Gamucci T, Pizzuti L, Sperduti I, et al. Neoadjuvant chemotherapy in triple-negative breast cancer: A multicentric retrospective observational study in real-life setting. J Cell Physiol 2018;233:2313-23.

21. Palmer JR, Ruiz-Narvaez EA, Rotimi CN, et al. Genetic susceptibility loci for subtypes of breast cancer in an African American population. Cancer Epidemiol Biomarkers Prev 2013;22:127-34.

22. Liedtke C, Mazouni C, Hess KR, et al. Response to neoadjuvant therapy and long-term survival in patients with triple-negative breast cancer. J Clin Oncol 2008;26:1275-81.

23. Chou HH, Kuo WL, Yu CC, et al. Impact of age on pathological complete response and locoregional recurrence in locally advanced breast cancer after neoadjuvant chemotherapy. Biomed J 2019;42:66-74. 
24. Denkert C, von Minckwitz G, Darb-Esfahani S, et al. Tumour-infiltrating lymphocytes and prognosis in different subtypes of breast cancer: a pooled analysis of 3771 patients treated with neoadjuvant therapy. Lancet Oncol 2018;19:40-50.

25. Nanda R, Liu MC, Yau C, et al. Pembrolizumab plus standard neoadjuvant therapy for high-risk breast cancer (BC): results from I-SPY 2. J Clin Oncol 2017;35:abstract 506.

26. Schmid P, Park YH, Muñoz-Couselo E, et al. Pembrolizumab (pembro) + chemotherapy (chemo) as neoadjuvant treatment for triple negative breast cancer (TNBC): preliminary results from KEYNOTE-173. J Clin Oncol 2017;35:abstract 556.

27. Gluz O, Nitz UA, Harbeck N, et al. Triple-negative highrisk breast cancer derives particular benefit from dose intensification of adjuvant chemotherapy: results of WSG AM-01 trial. Ann Oncol 2008;19:861-70.

28. Untch M, Fasching PA, Konecny GE, et al. PREPARE trial: a randomized phase III trial comparing preoperative, dose-dense, dose-intensified chemotherapy with epirubicin, paclitaxel and CMF versus a standard-dosed epirubicin/ cyclophosphamide followed by paclitaxel \pm darbepoetin alfa in primary breast cancer-results at the time of surgery. Ann Oncol 2011;22:1988-98.

29. von Minckwitz G, Schneeweiss A, Loibl S, et al. Neoadjuvant carboplatin in patients with triple-negative and HER2-positive early breast cancer (GeparSixto; GBG 66): a randomised phase 2 trial. Lancet Oncol 2014;15:747-56.

30. Untch M, Schneeweiss A, Salat C, et al. Long-term survival analysis of the randomized phase II trial investigating the addition of carboplatin to neoadjuvant therapy for triplenegative (TNBC) and HER2-positive early breast cancer (GeparSixto). Ann Oncol 2017;28:abstract 163PD.

31. Sikov WM, Berry DA, Perou CM, et al. Impact of the addition of carboplatin and/or bevacizumab to neoadjuvant once-per-week paclitaxel followed by dosedense doxorubicin and cyclophosphamide on pathologic complete response rates in stage II to III triple-negative

Cite this article as: de Paula BHR, Kumar S, Morosini FM, Calábria Cardoso DEM, de Sousa CAM, Crocamo S. Realworld assessment of the effect of impact of tumor size on pathological complete response rates in triple negative breast cancer after neoadjuvant chemotherapy. Chin Clin Oncol 2020;9(6):78. doi: 10.21037/cco-20-111 breast cancer: CALGB 40603 (Alliance). J Clin Oncol 2015;33:13-21.

32. Gerber B, Loibl S, Eidtmann H, et al. Neoadjuvant bevacizumab and anthracycline-taxane-based chemotherapy in 678 triple-negative primary breast cancers; results from the geparquinto study (GBG 44). Ann Oncol 2013;24:2978-84.

33. von Minckwitz G, Loibl S, Untch M, et al. Survival after neoadjuvant chemotherapy with or without bevacizumab or everolimus for HER2-negative primary breast cancer (GBG 44-GeparQuinto). Ann Oncol 2014;25:2363-72.

34. Earl HM, Hiller L, Dunn JA, et al. Efficacy of neoadjuvant bevacizumab added to docetaxel followed by fluorouracil, epirubicin, and cyclophosphamide, for women with HER2negative early breast cancer (ARTemis): an open-label, randomised, phase 3 trial. Lancet Oncol 2015;16:656-66.

35. Telli ML, Jensen KC, Vinayak S, et al. Phase II study of gemcitabine, carboplatin, and iniparib as neoadjuvant therapy for triple-negative and BRCA1/2 mutationassociated breast cancer with assessment of a tumor-based measure of genomic instability: PrECOG 0105. J Clin Oncol 2015;33:1895-901.

36. Rugo HS, Olopade OI, DeMichele A, et al. Adaptive Randomization of Veliparib-Carboplatin Treatment in Breast Cancer. N Engl J Med 2016;375:23-34.

37. Budman DR, Berry DA, Cirrincione CT, et al. Dose and dose intensity as determinants of outcome in the adjuvant treatment of breast cancer. J Natl Cancer Inst 1998;90:1205-11.

38. Wu K, Yang Q, Liu Y, et al. Meta-analysis on the association between pathologic complete response and triple-negative breast cancer after neoadjuvant chemotherapy. World J Surg Oncol 2014;12:95.

39. Partridge AH, Carey LA, Carey LA. Unmet Needs in Clinical Research in Breast Cancer: Where Do We Need to Go? Clin Cancer Res 2017;23:2611-6.

40. Place AE, Jin Huh S, Polyak K. The microenvironment in breast cancer progression: biology and implications for treatment. Breast Cancer Res 2011;13:227. 